\title{
Influence of hole transport material ionization energy on performance of perovskite solar cells.
}

Benedikt Dänekamp ${ }^{1}$, Nikolaos Droseros ${ }^{2}$, Demetra Tsokkou ${ }^{2}$, Verena Brehm ${ }^{1}$, Pablo P. Boix ${ }^{1 *}$, Michele Sessolo ${ }^{l}$, Natalie Banerji ${ }^{2}$, Henk J. Bolink ${ }^{l}$

${ }^{1}$ Instituto de Ciencia Molecular, Universidad de Valencia, C/ Catedrático J. Beltrán 2, 46980

Paterna (Valencia), Spain

${ }^{2}$ Department of Chemistry and Biochemistry, University of Bern, Freiestrasse 3, CH-3012 Bern, Switzerland

\begin{abstract}
Halide perovskites have shown excellent photophysical properties for solar cell applications which led to a rapid increase of the device efficiency. Understanding the charge carrier dynamics within the active perovskite absorber and at its interfaces will be key to further progress in their development. Here we present a series of fully evaporated devices employing hole transport materials with different ionization energies. The open circuit voltage of the devices, along with their ideality factors, confirm that the former is mainly determined by the bulk and surface recombination in the perovskite, rather than by the energetic offset between the valence band of the perovskite and the highest occupied molecular orbital of the organic transport layers. These results help to further understand the origin of the open circuit potential in perovskite solar cells, which is an important parameter that needs to be improved to further boost power conversion efficiencies.
\end{abstract}




\section{Introduction}

Perovskite solar cells are one of the most interesting alternatives to current photovoltaic technologies. Since the publication of the first solid state solar cells reports based on methylammonium lead iodide (MAPI) ${ }^{1,2}$, the development of the technology has resulted in a rapid increase of the device efficiencies, exceeding $22 \%^{3}$. Identifying the unique properties of halide perovskites is essential to understand the reasons for this remarkable progress. In this regard, unveiling the long charge carrier diffusion length $s^{4-6}$, sharp optical absorption edges ${ }^{7}$ and weak exciton binding energies ${ }^{8-10}$ are some of the key discoveries that contributed to explain the excellent device performances, as well as to gauge the full material potential.

However, there is still some debate regarding the main factors affecting the open circuit potential $\left(V_{o c}\right)$ of the devices ${ }^{11}$. In general, the splitting of electron and hole quasi-Fermi levels defines the maximum achievable $V_{o c}$ and it is determined by the charge generation/recombination rates along with the distribution of electronic states and charge carriers of the materials ${ }^{12}$. In multilayer perovskite solar cells, the problem shifts towards the role of the charge selective and transporting layers in contributing to the final $\mathrm{V}_{\text {oc }}$. Several reports have associated the high achieved $\mathrm{V}_{\text {oc }}$ to a high energy difference between the highest occupied molecular orbitals (HOMO) of the hole transport material (HTM) and the lowest unoccupied molecular orbitals (LUMO) of the electron transport material (ETM), together with a good alignment of these energy levels with the perovskite valence and conduction band, respectively ${ }^{13-18}$. Although some reports show an increase of the $\mathrm{V}_{\text {oc }}$ for HTMs with higher ionization energy $(\mathrm{IE})^{17,19,20}$, this trend is not necessarily reflected on the record performing perovskites solar cells, where best $\mathrm{V}_{\text {oc }}$ 's have been reported for both high HOMO (closer to the vacuum level) HTMs with well-aligned energy levels; and low HOMO (further from the vacuum level) HTMs with non-perfectly aligned energy levels ${ }^{21,22}$. More specifically, recent publications discuss the negligible $V_{\text {oc }}$ dependence on the ETM ${ }^{23}$ and HTM energy levels ${ }^{24}$. This view is also in line with previous results, where $\mathrm{V}_{\text {oc's }} \mathrm{s}$ higher than that expected from the HTM energy levels were obtained ${ }^{17}$. However, in all these cases, the HOMO of the employed HTM was close in energy to or just above the perovskite valence band maximum (reciprocally, the LUMO level of the ETM was aligned or slightly below the perovskite conduction band). 
Analyzing the effect of a set of HTMs with different HOMO energies appears as a straightforward approach to systematically investigate the relationship between the $V_{\text {oc }}$ and the energy levels of the extraction layers. However, it is important to note that the replacement of the HTM can also affect the interfacial charge recombination ${ }^{25}$, which has a large impact on the $V_{\text {oc. }}$ In addition, the large majority of the studies suffer from the limitations associated to solution-processed solar cells. This is in particular the case for $p$-i-n device architectures in which the holes are extracted through the front contact. In this configuration, the polar solutions containing the perovskite precursors are applied onto the apolar aromatic HTMs, leading to poor wetting and consequently poorly controlled film growth. Hence the resulting perovskite film is strongly affected by the surface of the $\mathrm{HTM}^{26}$, leading to different morphologies and, most likely, different trap densities. Moreover, most studies have been carried out on n-i-p solar cells, where the large majority of charge carriers is generated at the ETM/perovskite interface, which could reduce the effect of the HTM and underestimate its impact on the $\mathrm{V}_{\mathrm{oc}}{ }^{17,24}$.

a) Efrom $V L(e V)$

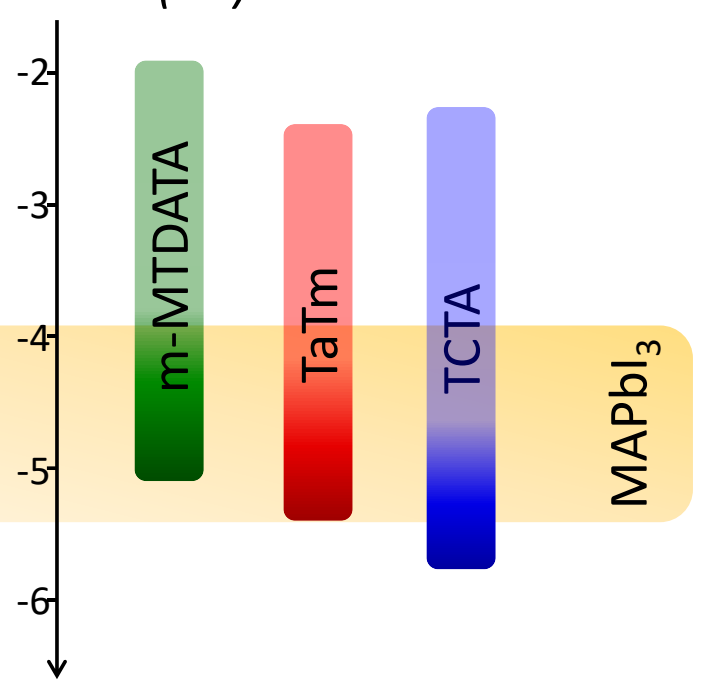

b)

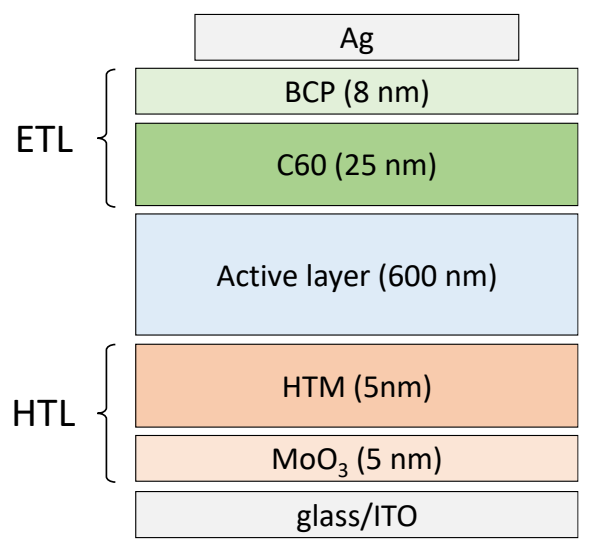

Figure 1. a) Energy diagram of different HTMs with respect to MAPI. The energy values of the HOMOs were determined by photoemission spectroscopy in air (PESA) (see supporting Information Figure S1). b) Scheme of completed stack with different HTMs. 
Here, we present a series of fully vacuum deposited perovskite solar cells with different HTMs. Vacuum deposition allows for the direct modification or substitution of any layer in the device stack with a negligible effect on the rest, as no solvents are used and hence no wetting or dewetting is occurring. In addition, the high voltages previously obtained with vacuum-deposited MAPI solar cells, which indicates the absence of non-radiative recombination paths at the selective interfaces, ${ }^{21}$ make it an ideal reference system to study interfacial modifications. Therefore, we are able to produce and study high efficiency perovskite solar cells based on a series of HTMs with more than $0.66 \mathrm{eV}$ difference in their IE, including materials with IE more than $0.3 \mathrm{eV}$ higher and lower than the perovskite valence band.

\section{Results and discussion}

The different vacuum-deposited materials used as HTMs are 4,4',4"-Tris[phenyl(mtolyl)amino]triphenylamine (m-MTDATA), N4,N4,N4",N4"'-tetra([1,1'-biphenyl]-4-yl)- [1,1':4',1"'terphenyl]-4,4"-diamine (TaTm) and Tris(4-carbazoyl-9-ylphenyl)amine (TcTa). Their respective ionization energies of $5.0 \mathrm{eV}, 4.4 \mathrm{eV}, 5.7 \mathrm{eV}$ (Figure 1a) were measured using photo-electron spectroscopy in air (see supporting Information Figure S1). The solar cells were fabricated following a p-i-n architecture consisting of pre-patterned ITO on glass with ITO, $\mathrm{MoO}_{3}, \mathrm{HTM}$, MAPI, fullerene $\left(\mathrm{C}_{60}\right)$, 2,9-Dimethyl-4,7-diphenyl-1,10-phenanthroline $(\mathrm{BCP})$ and $\mathrm{Ag}$ as a top contact (Figure 1b). Detailed experimental conditions can be found in the Supplementary Information. Using a p-i-n device structure ensures that the majority of carriers are generated at the HTM/perovskite interface. Representative current-voltage characteristics measured under $100 \mathrm{~mW} \mathrm{~cm}^{-2}$ illumination in forward and reverse bias are displayed in Figure 2a, whereas the corresponding photovoltaic parameters with the associated statistical error ( $>10$ samples per parameter) can be found in Table 1. Slight current-voltage hysteresis is only visible in the TcTa based device, while it is negligible in the other cases. Devices based on TaTm show the highest current, $20.1 \mathrm{~mA} \mathrm{~cm}^{-2}$, in contrast to the ones with TcTa and m-MTDATA, which are $1.2 \mathrm{~mA} \mathrm{~cm}^{-2}$ lower. The FF of TaTm and m-MTDATA based devices are comparable $(76 \%$ and $75 \%$ 
respectively), with a lower value (64\%) obtained for the solar cells employing TcTa. More interestingly, devices with m-MTDATA and TcTa show almost no difference in $\mathrm{V}_{\text {oc }}(1.008 \mathrm{~V}$ and $1.000 \mathrm{~V}$ ) yet with an IE difference as large as $0.66 \mathrm{eV}$ among them. Devices with TaTm present the highest $V_{o c}$ of $1060 \mathrm{mV}$. Since these results do not show any evident trend between the HTMs energetics and the resulting $\mathrm{V}_{\text {oc }}$, further studies were carried out to elucidate the relationship among those parameters.

The dominant type of recombination was investigated by measuring the $\mathrm{V}_{\text {oc }}$ as a function of light intensity ${ }^{27}$. Figure 3 shows the light intensity dependent $V_{o c}$ and ideality factor for devices with different HTMs. The $\mathrm{V}_{\text {oc }}$ depends logarithmically on the light intensity and introducing the ideality factor $n_{\mathrm{ID}}$ as a pre-factor we find ${ }^{27}$ :

$e V_{o c}=E_{g}-n_{I D} k_{B} T \ln \frac{I_{0}}{I}$

with $E_{g}$ being the bandgap, $T$ the temperature, $\mathrm{k}_{\mathrm{B}}$ the Boltzmann constant and $\mathrm{I}_{0}$ the reverse saturation current. Measuring the $\mathrm{V}_{\mathrm{oc}}$ as a function of light intensity for devices with varying HTMs results in ideality factors of $\sim 1.7$ for TaTm and $\sim 1.2$ for TcTa and m-MTDATA. Ideality factors close to one can be attributed to direct recombination, which is usually interpreted as non-radiative surface recombination (if the associated $V_{o c}$ is low compared to the absorber's band gap) or to radiative bulk recombination (in case of high $\mathrm{V}_{\mathrm{oc}}$ 's). Ideality factors close to 2 are usually associated to non-radiative SRH recombination. Therefore, against the common believe, low ideality factors are not necessarily beneficial. In fact several perovskite solar cells with low ideality factors and low $V_{\text {oc }}$ have been reported ${ }^{11,28,29}$.

Considering this, devices using TaTm show higher $\mathrm{V}_{\text {oc }}$ and an ideality factor of 1.7, while the solar cells with TcTa and m-MTDATA have lower $V_{\text {oc }}$ and an ideality factor of 1.2. This points towards an enhanced surface recombination which limits the $V_{\text {oc }}$ of the TcTa and m-MTDATA devices, while the $\mathrm{V}_{\text {oc }}$ of TaTm is mainly determined by SRH-recombination. The origin of the larger surface recombination at the interface between MAPI and TcTa or m-MTDATA is not clear yet. The IE of the HTM itself is unlikely to influence the splitting of the quasi-Fermi levels, in particular the position of the holes' quasi-Fermi level $\left(E_{F h}\right)$, which is determined by the absorber. This is justified by the low exciton binding energy ${ }^{30}$ and small dark charge carrier density ${ }^{31}$ of MAPI, and it is in 
line with previous observations where modifications of the HTM and ETM energetics did not affect the photovoltage ${ }^{23,24}$. However, our results also suggest that it is possible using HTMs with a HOMO lower than the absorber's CB without a significant voltage penalty associated to the energetics.

It is thus important to discuss the implications of these HTMs in the other relevant photovoltaic parameters of the devices.
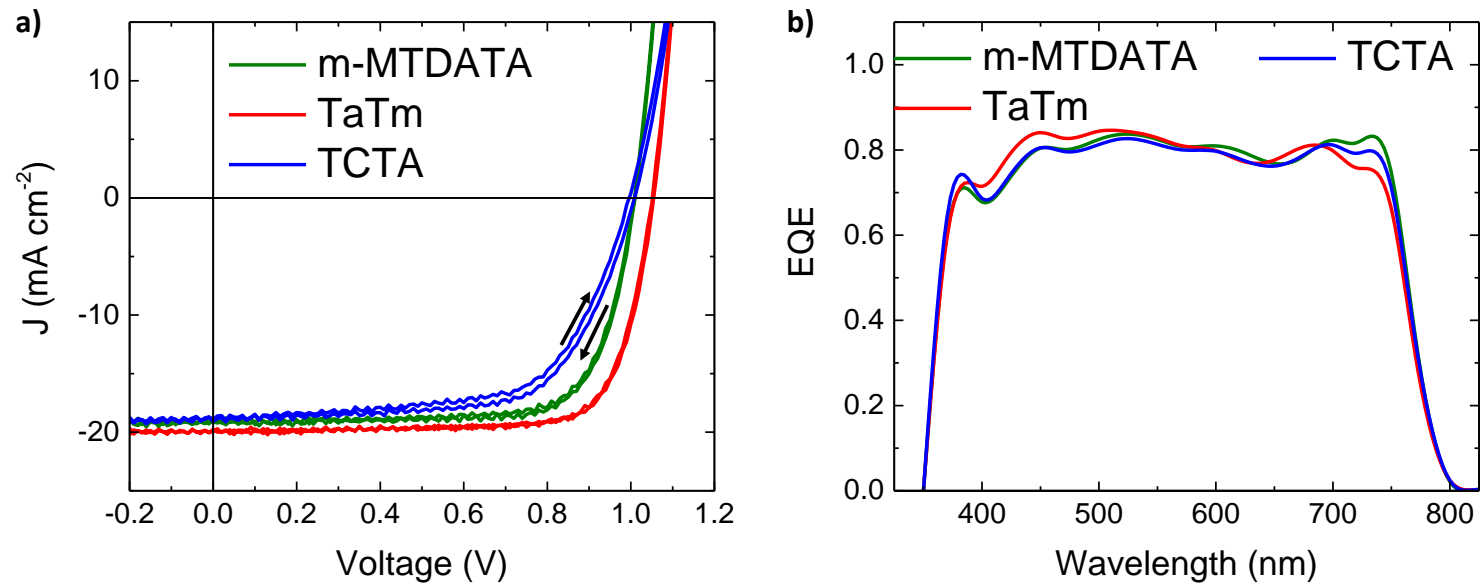

Figure 2. (a) J-V curves under $100 \mathrm{~mW} \mathrm{~cm}^{-2}$ illumination in forward and reverse bias and (b) EQE spectra of representative perovskite solar cells produced with different $5 \mathrm{~nm}$ thick HTMs.

Table 1. IPs of different HTMs and representative photovoltaic parameters obtained when implemented in solar cells.

\begin{tabular}{|c|c|c|c|c|c|}
\hline HTM & $\mathrm{IP}(\mathrm{eV})$ & PCE (\%) & $\mathrm{V}_{\mathrm{oc}}(\mathrm{mV})$ & $\mathrm{J}_{\mathrm{sc}}\left(\mathrm{mA} / \mathrm{cm}^{2}\right)$ & $\mathrm{FF}$ \\
\hline m-MTDATA & 5.0 & $14.4 \pm 1.2$ & $1008 \pm 4$ & $18.9 \pm 0.5$ & $75 \pm 3$ \\
\hline TaTm & 4.4 & $16.3 \pm 0.8$ & $1060 \pm 6$ & $20.1 \pm 0.8$ & $76 \pm 1$ \\
\hline TсTa & 5.7 & $12.2 \pm 1.6$ & $1000 \pm 8$ & $18.9 \pm 1.1$ & $64 \pm 9$ \\
\hline
\end{tabular}




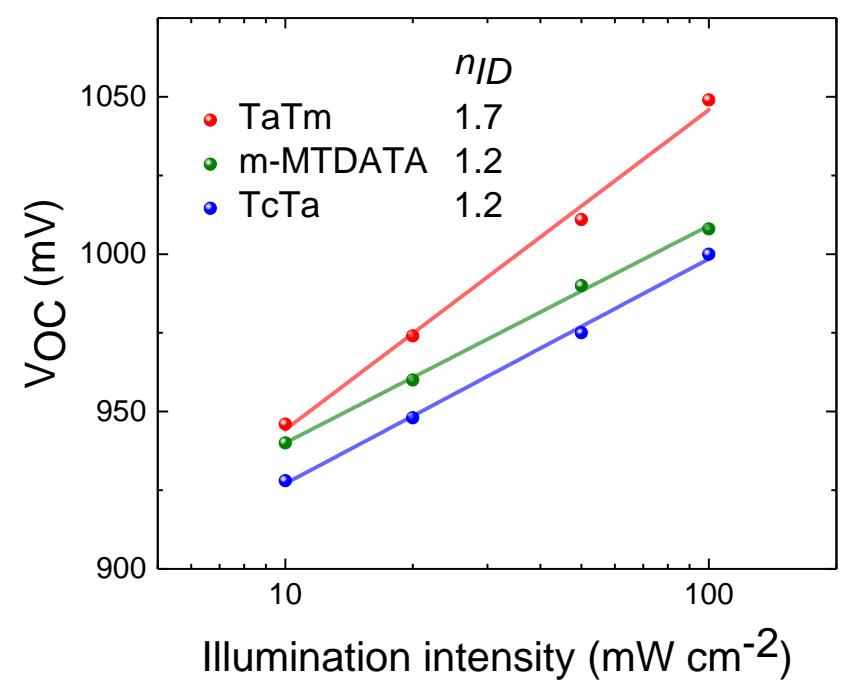

Figure 3. Light intensity dependent $\mathrm{V}_{\text {oc }}$ and extracted ideality factors for perovskite solar cells with different HTMs.

The three solar cells mainly differ in FF, with TaTm and m-MTDATA having high values of around 75 and TcTa having a lower FF of 64. This can be attributed to a higher series resistance of TcTa, which is also reflected in the dark IV-curve above around 1V (Figure S2). All devices reach relatively high short circuit currents, $20.1 \mathrm{~mA} / \mathrm{cm}^{2}$ for TaTm and $18.9 \mathrm{~mA} / \mathrm{cm}^{2}$ for TcTa and mMTDATA. The EQE curves shown in Figure $2 \mathrm{~b}$ are very similar which is consistent with the results discussed before. This point highlights the good extraction of the photogenerated charge obtained with both TcTa and m-MTDATA despite their HOMO level mismatch with the perovskite valence band. To confirm this point, the charge injection from MAPI to the HTMs was studied using femtosecond transient absorption spectroscopy (TAS). Figure 4 shows the time-resolved transient absorption dynamics for perovskite/HTM double layers. Samples were excited with laser pulses at $600 \mathrm{~nm}$ with a constant excitation density of $1.4 \cdot 10^{17} \mathrm{~cm}^{-3}$, and probed at the maximum of the perovskite ground state bleaching (GSB) band. An instantaneously reduced signal in the perovskite GSB is observed upon the addition of the HTMs, indicating that the hole injection takes place for all HTMs, even faster than the time resolution of the setup of about 60 
fs. The TA spectra at different time delays after excitation for the perovskite/TaTm bilayer are shown in Figure S5. A detailed photophysical study of the systems will be published subsequently.

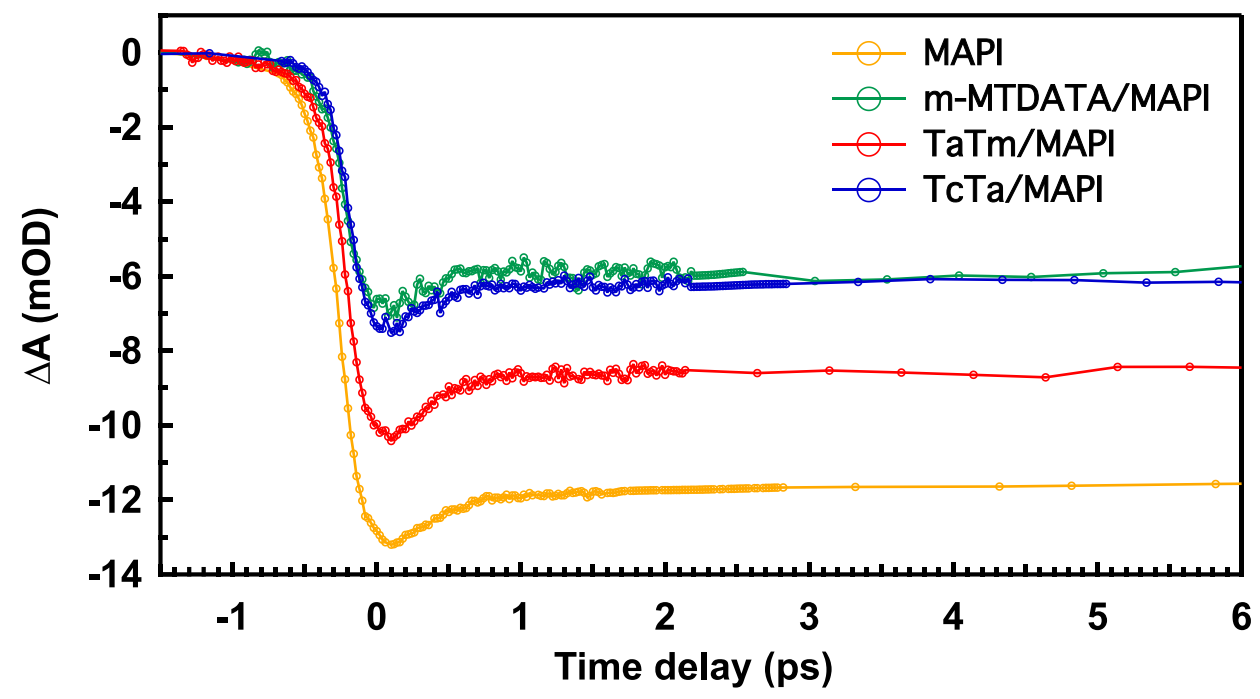

Figure 4. Time-resolved transient absorption dynamics for neat MAPI and the perovskite/HTM double layers, excited at $600 \mathrm{~nm}$ (at constant excitation density of $1.4 \cdot 10^{17} \mathrm{~cm}^{-3}$ ) and probed at the perovskite maximum ground state bleaching band.

\section{Conclusions}

In summary, we produced a stack of fully evaporated devices based on different HTMs with an IP-difference as high as $0.66 \mathrm{eV}$ among them. Devices produced with these HTMs showed Voc's ranging from $1000 \mathrm{mV}(\mathrm{TcTa}$,$) to 1060 \mathrm{mV}$ (TaTm). The measurement of the respective ideality factors allows us to explain the differences in $V_{O C}$ by the nature of the recombination processes. These results support that the $V_{0 c}$ of perovskite solar cells is not necessarily limited by the energetics of the hole transporting material, but mainly by the different recombination paths. In addition, we show efficient charge extraction by HTMs with misaligned HOMO with the perovskite ( $>300 \mathrm{meV}$ above and below the perovskite's valence band). These results point towards HTM designing routes which can improve the performance of perovskite solar cells, focusing on the interfacial recombination reduction rather than on the energetic alignment. 


\section{Acknowledgements}

We acknowledge financial support from the European Union H2020 project INFORM (grant 675867). N.D. D.T. and N.B. would like to acknowledge the University of Bern, Switzerland, for providing funding and infrastructure. Financial support is acknowledged from the Spanish Ministry of Economy and Competitiveness (MINECO) via the Unidad de Excelencia María de Maeztu MDM-2015-0538, MAT2017-88821-R, PCIN-2015-255, PCIN-2017-014 and the Generalitat Valenciana (Prometeo/2016/135). P.B and M.S. thanks the MINECO for their RyC contracts. P.B. acknowledges the financial support from the Conselleria d'Educació, Investigació, Cultura i Esport Valenciana (SEJI2017/2017/012).

\section{References}

1. Michael M. Lee. Efficient Hybrid Solar Cells Based on Meso-Superstructured Organometal Halide Perovskites. Science (80- ). 2012;338(6107):643-647. doi:10.1126/science.1228604.

2. Kim H-S, Lee C-R, Im J-H, et al. Lead lodide Perovskite Sensitized All-Solid-State Submicron Thin Film Mesoscopic Solar Cell with Efficiency Exceeding 9\%. Sci Rep. 2012;2:591. doi:10.1038/srep00591.

3. Yang WS, Park B-W, Jung EH, et al. lodide management in formamidinium-lead-halidebased perovskite layers for efficient solar cells. Science (80- ). 2017;356(6345):13761379. doi:10.1126/science.aan2301.

4. Nie W, Tsai H, Asadpour R, et al. High-efficiency solution-processed perovskite solar cells with millimeter-scale grains. Science (80- ). 2015;347(6221):522 LP-525. doi:10.1126/science.aaa0472.

5. Shi D, Adinolfi V, Comin R, et al. Low Trap-State Density and Long Carrier Diffusion in Organolead Trihalide Perovskite Single Crystals. Sci (80- ). 2015;347(6221):519-522. 
doi:10.1126/science.aaa2725.

6. Xing G, Mathews N, Sun S, et al. Long-Range Balanced Electron- and Hole-Transport Lengths in Organic-Inorganic CH3NH3PbI3. Science (80- ). 2013;342(6156):344-347. doi:10.1126/science.1243167.

7. De Wolf S, Holovsky J, Moon SJ, et al. Organometallic halide perovskites: Sharp optical absorption edge and its relation to photovoltaic performance. J Phys Chem Lett. 2014;5(6):1035-1039. doi:10.1021/jz500279b.

8. Lin Q, Armin A, Nagiri RCR, Burn PL, Meredith P. Electro-optics of perovskite solar cells. Nat Photonics. 2015;9(2):106-112. doi:10.1038/nphoton.2014.284.

9. Savenije TJ, Ponseca CS, Kunneman L, et al. Thermally activated exciton dissociation and recombination control the carrier dynamics in organometal halide perovskite. J Phys Chem Lett. 2014;5(13):2189-2194. doi:10.1021/jz500858a.

10. Yang Y, Yang M, Li Z, Crisp R, Zhu K, Beard MC. Comparison of Recombination Dynamics in $\mathrm{CH} 3 \mathrm{NH} 3 \mathrm{PbBr} 3$ and $\mathrm{CH} 3 \mathrm{NH} 3 \mathrm{PbI} 3$ Perovskite Films: Influence of Exciton Binding Energy. J Phys Chem Lett. 2015;6(23):4688-4692. doi:10.1021/acs.jpclett.5b02290.

11. Tress W. Perovskite Solar Cells on the Way to Their Radiative Efficiency Limit - Insights Into a Success Story of High Open-Circuit Voltage and Low Recombination. Adv Energy Mater. 2017. doi:10.1002/aenm.201602358.

12. Shao Y, Yuan Y, Huang J. Correlation of energy disorder and open-circuit voltage in hybrid perovskite solar cells. Nat Energy. 2016;1(1):1-9. doi:10.1038/nenergy.2015.1.

13. Song $Y$, Lv S, Liu X, et al. Energy level tuning of TPB -based hole-transporting materials for highly efficient perovskite solar cells. Chem Commun. 2014;50(96):15239-15242. doi:10.1039/c4cc06493c.

14. Cheng $M, X u B, C h e n ~ C$, et al. Phenoxazine-based small molecule material for efficient perovskite solar cells and bulk heterojunction organic solar cells. Adv Energy Mater. 2015;5(8):1-9. doi:10.1002/aenm.201401720. 
15. Kim G-W, Kang G, Kim J, et al. Dopant-free polymeric hole transport materials for highly efficient and stable perovskite solar cells. Energy Environ Sci. 2016;9(7):2326-2333. doi:10.1039/C6EE00709K.

16. Lim K, Kim H, Jeong J, Kim H, Kim JY, Lee T. Boosting the Power Conversion Effi ciency of Perovskite Solar Cells Using Self-Organized Polymeric Hole Extraction Layers with High Work Function. Adv Mater. 2014;26(37):1-6. doi:10.1002/adma.201401775.

17. Ryu S, Noh JH, Jeon NJ, et al. Voltage output of efficient perovskite solar cells with high open-circuit voltage and fill factor. Energy Environ Sci. 2014;7(8):2614-2618. doi:10.1039/c4ee00762j.

18. Zhang J, Xu B, Johansson MB, et al. Strategy to Boost the Efficiency of Mixed-Ion Perovskite Solar Cells: Changing Geometry of the Hole Transporting Material. ACS Nano. 2016;10(7):6816-6825. doi:10.1021/acsnano.6b02442.

19. Polander LE, Pahner P, Schwarze M, Saalfrank M, Koerner C, Leo K. Hole-transport material variation in fully vacuum deposited perovskite solar cells. APL Mater. 2014;2(8):081503. doi:10.1063/1.4889843.

20. Kulbak M, Cahen D, Hodes G. How Important Is the Organic Part of Lead Halide Perovskite Photovoltaic Cells? Efficient CsPbBr 3 Cells. J Phys Chem Lett. 2015;6(13):2452-2456. doi:10.1021/acs.jpclett.5b00968.

21. Pérez-del-Rey D, Boix PP, Sessolo M, Hadipour A, Bolink HJ. Interfacial Modification for High-Efficiency Vapor-Phase-Deposited Perovskite Solar Cells Based on a Metal Oxide Buffer Layer. J Phys Chem Lett. 2018:1041-1046. doi:10.1021/acs.jpclett.7b03361.

22. Yu W, Yu S, Zhang J, et al. Two-in-one additive-engineering strategy for improved air stability of planar perovskite solar cells. Nano Energy. 2018;45(October 2017):229-235. doi:10.1016/j.nanoen.2017.12.041.

23. Ravishankar S, Gharibzadeh S, Roldán-Carmona C, et al. Influence of Charge Transport Layers on Open-Circuit Voltage and Hysteresis in Perovskite Solar Cells. Joule. 
2018;2(4):788-798. doi:10.1016/j.joule.2018.02.013.

24. Belisle RA, Jain P, Prasanna R, Leijtens T, McGehee MD. Minimal Effect of the HoleTransport Material Ionization Potential on the Open-Circuit Voltage of Perovskite Solar Cells. ACS Energy Lett. 2016;1(3):556-560. doi:10.1021/acsenergylett.6b00270.

25. Yamada Y, Yamada T, Shimazaki A, Wakamiya A, Kanemitsu Y. Interfacial Charge-Carrier Trapping in CH3NH3PbI3 -Based Heterolayered Structures Revealed by Time-Resolved Photoluminescence Spectroscopy. J Phys Chem Lett. May 2016:acs.jpclett.6b00653. http://dx.doi.org/10.1021/acs.jpclett.6b00653. Accessed May 9, 2016.

26. McMeekin DP, Wang Z, Rehman W, et al. Crystallization Kinetics and Morphology Control of Formamidinium-Cesium Mixed-Cation Lead Mixed-Halide Perovskite via Tunability of the Colloidal Precursor Solution. Adv Mater. 2017;29(29). doi:10.1002/adma.201607039.

27. Tress W, Yavari M, Domanski K, et al. Interpretation and Evolution of Open-Circuit Voltage, Recombination, Ideality Factor and Subgap Defect States during Reversible Light-Soaking and Irreversible Degradation of Perovskite Solar Cells. Energy Environ Sci. 2017;11:151-165. doi:10.1039/C7EE02415K.

28. Miyano K, Yanagida M, Tripathi N, Shirai Y. Simple characterization of electronic processes in perovskite photovoltaic cells. Appl Phys Lett. 2015;106(9):093903. http://scitation.aip.org/content/aip/journal/apl/106/9/10.1063/1.4914086. Accessed March 5, 2015.

29. Miyano K, Tripathi N, Yanagida M, Shirai Y. Lead Halide Perovskite Photovoltaic as a Model p-i-n Diode. Acc Chem Res. 2016;49(2):303-310. http://dx.doi.org/10.1021/acs.accounts.5b00436. Accessed March 10, 2016.

30. Miyata A, Mitioglu A, Plochocka P, et al. Direct measurement of the exciton binding energy and effective masses for charge carriers in organic-inorganic tri-halide perovskites. Nat Phys. 2015;11:582-587. doi:10.1038/nphys3357.

31. Chen Y, Yi HT, Wu X, et al. Extended carrier lifetimes and diffusion in hybrid perovskites 
revealed by Hall effect and photoconductivity measurements. Nat Commun. 2016;7:12253. doi:10.1038/ncomms12253. 